BNL-75097-2005-CP

\title{
Effect of Te precipitates on the performance of CdZnTe detectors
}

\author{
G.A. Carini ${ }^{1,2}$, A.E. Bolotnikov ${ }^{1}$, G.S. Camarda ${ }^{1}$, G.W. Wright ${ }^{1}$, \\ L. $\mathbf{L i}^{3}$, and R.B. James ${ }^{1}$ \\ ${ }^{1}$ Department of Nonproliferation and National Security \\ Brookhaven National Laboratory, Upton, New York 11973-5000 \\ ${ }^{2}$ Department of Electrical Engineering \\ University of Palermo, Palermo, 90128 Italy \\ ${ }^{3}$ Yinnel Tech, Inc., South Bend, Indiana 46619
}

Presented at the 2005 US Workshop on the Physics and Chemistry of II-VI Materials

Boston, Massachusetts

September 20-22, 2005

\author{
Nonproliferation and National Security Department \\ Brookhaven National Laboratory \\ P.O. Box 5000 \\ Upton, NY 11973-5000 \\ www.bnl.gov
}

\begin{abstract}
Notice: This manuscript has been authored by employees of Brookhaven Science Associates, LLC under Contract No. DE-AC02-98CH10886 with the U.S. Department of Energy. The publisher by accepting the manuscript for publication acknowledges that the United States Government retains a non-exclusive, paid-up, irrevocable, world-wide license to publish or reproduce the published form of this manuscript, or allow others to do so, for United States Government purposes.
\end{abstract}

This preprint is intended for publication in a journal or proceedings. Since changes may be made before publication, it may not be cited or reproduced without the author's permission. 


\section{DISCLAIMER}

This report was prepared as an account of work sponsored by an agency of the United States Government. Neither the United States Government nor any agency thereof, nor any of their employees, nor any of their contractors, subcontractors, or their employees, makes any warranty, express or implied, or assumes any legal liability or responsibility for the accuracy, completeness, or any third party's use or the results of such use of any information, apparatus, product, or process disclosed, or represents that its use would not infringe privately owned rights. Reference herein to any specific commercial product, process, or service by trade name, trademark, manufacturer, or otherwise, does not necessarily constitute or imply its endorsement, recommendation, or favoring by the United States Government or any agency thereof or its contractors or subcontractors. The views and opinions of authors expressed herein do not necessarily state or reflect those of the United States Government or any agency thereof. 


\title{
Effect of Te precipitates on the performance of CdZnTe detectors
}

\author{
G. A. Carini ${ }^{1,2^{*}}$, A. E. Bolotnikov ${ }^{1}$, G. S. Camarda ${ }^{1}$, G. W. Wright ${ }^{1}$, L. $\mathrm{Li}^{3}$, and R. B. James ${ }^{1}$ \\ ${ }^{1}$ Department of Nonproliferation and National Security \\ Brookhaven National Laboratory, Upton, New York 11973-5000 \\ ${ }^{2}$ Department of Electrical Engineering \\ University of Palermo, Palermo, 90128 Italy \\ ${ }^{3}$ Yinnel Tech, Inc., South Bend, Indiana 46619
}

\begin{abstract}
A recent study of long-drift CdZnTe (CZT) Frisch-ring detectors showed that fluctuations of the collected charge (and device response) depend on the device dimensions and the concentration of Te precipitates in the material. This observation, which could be explained as the cumulative effect of precipitates, led to the investigation of thin $(1 \mathrm{~mm})$ planar detectors, where the effects of precipitates can be more clearly ascertained. To perform the investigation, a measurement facility was developed that allowed for high-resolution spatial mapping of the performance of CZT devices. New measurements emerging from this facility provided the first detailed comparisons of the micro-scale X-ray maps and infrared microscopy images for thin CZT samples. Analysis of the data showed conclusively that local deteriorations of device response fully correlate with Te precipitates seen in the IR images. Effects of surface processing conditions on the detector response were also clearly observed.
\end{abstract}

*carini@bnl.gov; carini@diepa.unipa.it; phone: 631344 3004; fax: 6313443374 
The role of Te inclusions and precipitates has been under discussion since the introduction of the CZT detectors as a room-temperature X-and gamma-ray detector [1]. Inclusions originate at the growth interface: Te-rich droplets are trapped at the boundary layer of the interface as a consequence of morphological instability [2,3]. Inclusions are also distributed throughout the single-crystal material. Te precipitates are a consequence of the retrograde solid solubility in the CdTe binary system. Twins and grain boundaries are preferential nucleation sites, and high densities of Te precipitates are found all along the boundaries. In this letter we define precipitates as any volume of Te material with a size smaller than $30 \mu \mathrm{m}$, which is trapped inside the crystal [4].

Te-rich volumes have different optical and electrical properties than bulk CZT. Due to the different absorbance, they can be identified by IR transmission microscopy and microspectroscopy. Due to the narrow band-gap of Te $(\sim 0.3 \mathrm{eV})$, Te precipitates have higher electrical conductivity, which may affect the detector leakage current and distort the electric field distribution and carrier transport in CZT devices. When a crystal shows a high density of Te precipitates (e.g., along grain boundaries and twins) extending from one electrode to another, it results in a very high leakage current and poor spectrometric performance throughout the region with clusters of Te precipitates. These measurements created new interest in the development of improved crystal-growth methods capable of producing large CZT single crystals for fabrication of high-quality gamma spectrometers. Despite the new thrust by many organizations to avoid grain boundaries and twins in the selection of CZT blanks, the role of dispersed Te precipitates within the single-crystal CZT volumes was still unclear, because the techniques used to map the variability of X-ray detector response as a function of location probed the material on a length scale of hundreds of microns; thus, they were unable to precisely measure the effects of either a single Te precipitate or other isolated small microstructural defects with sizes of 1020 microns or smaller [5]. By developing a unique measurement facility capable of an order-ofmagnitude improvement of the spatial resolution, this study was able to provide unequivocal evidence correlating regions with degraded detector performance with the presence of dispersed precipitates.

In this letter the correlation between Te precipitates, which are visible in the IR images of CZT crystals, and the deterioration of device response was measured for a 11-mm long Frisch-ring and a 1$\mathrm{mm}$ thick planar detector. These devices were chosen to measure the correlations on large (cumulative effect of a large number of precipitates) and local scales (effect of individual precipitates). The amplitude of the output signal, measured for a Frisch-ring device, is proportional to the total collected charge only. Hence, it provides a perfect way to study the cumulative effect of precipitates versus the 
depth of interaction. To study the local variation of charge collection caused by a single inclusion, the probability of superposing effects from two of more precipitates located along the same line of an electron cloud's trajectory must be excluded. This can be achieved by using thin planar detectors. Moreover, it is known that precipitates are surrounded by regions with high concentration of dislocations with many defect levels. In addition, the space charge, accumulated by trapping at these levels, can distort the local electric field. As a result, the electron cloud trajectories are probably not straight lines connecting the two electrodes, so that the X-ray map measured for thicker devices will become more distorted than for thinner detectors.

Several $\sim 5 \times 5 \times 10 \mathrm{~mm}^{3}$ Frisch-ring [6,7] and planar detectors were studied in this work. The original CZT crystals, grown by Modified Vertical Bridgman method, were provided by Yinnel Tech., Inc. During the measurements, the detectors were mounted inside a standard device holder, purchased from eV-Products, and irradiated from the cathode side by photons from either a synchrotron radiation source or radioactive isotopes. The output signals were readout and processed using standard spectroscopy electronics, including a charge-sensitive preamplifier, a shaper, and a MCA card to collect pulse-height spectra. To study the local variation of devices response, X-ray scans were performed at the National Synchrotron Light Source (NSLS) by using beams with different sizes; the smallest was slightly less than $10 \times 10 \mu \mathrm{m}^{2}$. An $85-\mathrm{keV}$ quasi-monochromatic beam with a $10-\mathrm{keV}$ spread (white beam mode with attenuator) and a $30-\mathrm{keV}$ monochromatic beam were used. The absorption lengths of 30 and $85 \mathrm{keV}$ photons in CZT are 0.3 and $3 \mathrm{~mm}$, respectively, which means that the $30-\mathrm{keV}$ beam is much more sensitive to surface defects. For each $\mathrm{X}-\mathrm{Y}$ position of the X-ray beam, the pulse-height spectra were collected, and the peak positions, which are proportional to the total collected charge, were evaluated by using a Gaussian fit. The scan results were plotted as twodimensional maps of device responses.

Fig. 1 shows the X-ray maps and pulse-high spectra measured for 11-mm and 16-mm long Frisch-ring CZT detectors. Both detectors were fabricated from the same region of a CZT ingot, and they had similar $\mu \tilde{\tau}$ products; the devices displayed much different spectroscopic performance. The IR analysis of the crystals used to produce the detectors showed a lower concentration of dispersed precipitates for the detector with better performance (shorter one in this case). This suggests that the degradation of the device response (peak broadening without the significant shift in the peak position) could be attributed to the fluctuations of charge loss caused by the trapping centers associated with precipitates. These fluctuations are also reflected in the X-ray scans carried out for these detectors. 
However, the pulse-height spectra and X-ray maps, like the examples shown in Fig. 1, can only demonstrate the cumulative effect of precipitates and provide only indirect evidence of their role. To prove that precipitates indeed affect the charge collection efficiency in CZT devices and are responsible for the degraded energy spectra, several $\sim 1$-mm thick CZT devices were investigated with the goal to measure the correlations between Te precipitates locations, as seen with the IR transmission microscope, and the device response, as identified with X-ray mapping (Fig. 2). The 1$\mathrm{mm}$ thick detectors were chosen to minimize the effects of multiple precipitates overlapping the trajectory of the electron clouds and image distortions due to electric field non-uniformities created from clusters of precipitates. As seen in the IR micrographs and x-ray scans, the Te precipitates in the IR images correspond to the "dark" spots (where the device response drops off up to $50 \%$ of its average value) in the X-ray maps. Several similar measurements for different thin devices fabricated from CZT crystals grown by different techniques showed for the first time a $100 \%$ correlation between the locations of precipitates and the areas with poor detector performance.

Te precipitates (including possibly the areas of very high dislocations surrounding the precipitates) act like electron traps, but they are different from the single-level traps normally present in CZT material. The latter are uniformly distributed over a device volume and continuously trap electrons as the electron cloud drifts from the point of interaction toward the anode. In this case, the charge loss depends on the electron cloud's drift time, but the fluctuations in the lost charge are somewhat negligible because of the large number of the point defects distributed throughout the detector volume. Nevertheless, the single-level traps still result in peak shifts and broadening, because the electron cloud's drift length varies as a function of the locations of the interaction points. Since the peak shift and broadening for a large number of randomly distributed defects can be easily corrected by applying depth sensing or rise-time correction techniques, they are not the primary material factor limiting the performance of CZT detectors.

In contrast to randomly distributed single-level traps, precipitates can be considered as extended local defects with a very high local concentration of trapping centers. In this case, an unpredictable amount of charges will be trapped, and the amount of trapping cannot be corrected with current techniques. Here, the fluctuations in the charge loss become proportional to the total number of such defects encountered by the electron cloud. Moreover, any electric-field distortions around these defects can also contribute to dispersion of the collected charges and to degradation in the spectroscopic performance. Fig. 3 shows the results of the X-ray scan plotted in 3D coordinates. The 
flat surface represents the averaged level of the device response, and the many "wells" in the figure correspond to the locations of the precipitates in CZT. In the past, X-ray, gamma-ray, and alphaparticle scans of CZT detectors were performed to investigate the uniformity of device response [8,9], but the size of the beams used in those measurements were $100 \mu \mathrm{m}$ or larger. It turned out that the size of the beam used for the scans is an important factor, because it limits the ability to spatially resolve the effects of isolated detectable precipitates. Fig. 4 presents four maps of the same device area evaluated with different spatial resolution to simulate different beam sizes: 10x10, 20x20, 100x100, and $200 \times 200 \mu \mathrm{m}^{2}$. The original X-ray map was measured with $10-\mu \mathrm{m}$ steps in both directions with the beam size of $10 \times 10 \mu \mathrm{m}^{2}$. To generate the other maps, the pulse-height spectra measured for several adjacent beam locations $(2 \times 2,4 \times 4$, etc.) were added together and new peak positions were determined by using a Gaussian fit. This procedure allowed us to accurately simulate larger beam sizes. No precipitates are observed at $100 \times 100 \mu \mathrm{m}^{2}$ beam size, and the crystal seems fairly uniform in the 100 $\mu \mathrm{m}$ map. The prior mapping measurements using larger beam sizes conducted by many groups caused the CZT detector community to incorrectly assume that isolated Te precipitates did not adversely affect detector quality. One possibly justification for this premise was that the electric field was modified around isolated precipitates in such a way that the electron and hole clouds were steered around the precipitates, thereby producing only a minor contribution to carrier trapping. The new measurements offered in this paper allow one to easily discern the effects of precipitates on electron trapping and elucidate the critical need to address the presence of isolated precipitates and precipitates within single-crystal CZT detectors.

Finally, from the results of these studies, we see that defects close to the cathode widely affect the local gamma response when the $30-\mathrm{keV}$ beam was used. However, when an $85-\mathrm{keV}$ beam was used, defects positioned deeper in the crystal became more important.

In conclusion, Te precipitates located in the IR images fully correlate with the deterioration of the X-ray and gamma spectroscopic response of detectors by producing degraded localized zones within the devices. This understanding will spawn new thrusts among the CZT nuclear detector community to conduct growth experiments designed to minimize the presence of dispersed Te precipitates within single-crystal CZT without adversely affecting the bulk electrical resisitivity or creating new trapping centers.

Further investigations of the local crystal properties (e.g., mobility, drift time, and waveform analysis) will be reported in the future. 
The authors acknowledge the support of the technical and scientific staff at Brookhaven National Laboratory's NSLS. Special thanks go to Peter Siddons and Claudio Arnone. 
Figures.

(a)

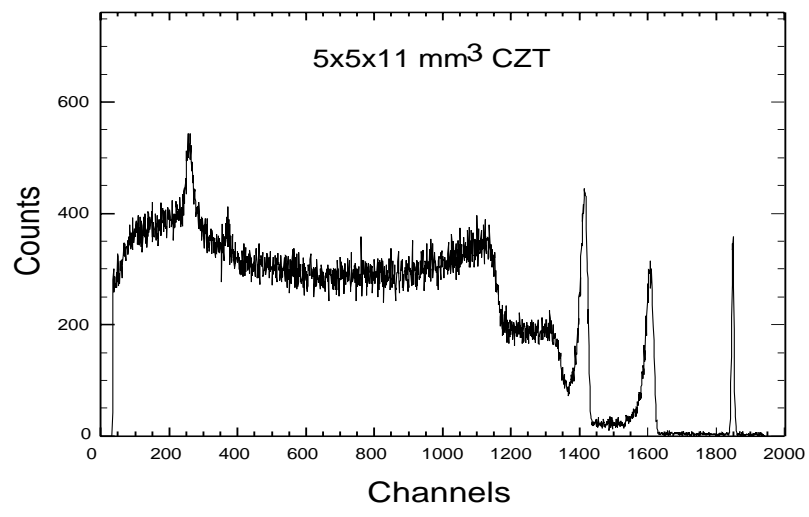

$X$-ray map

$5 \times 5 \times 11 \mathrm{~mm}^{3} \mathrm{CZT}$

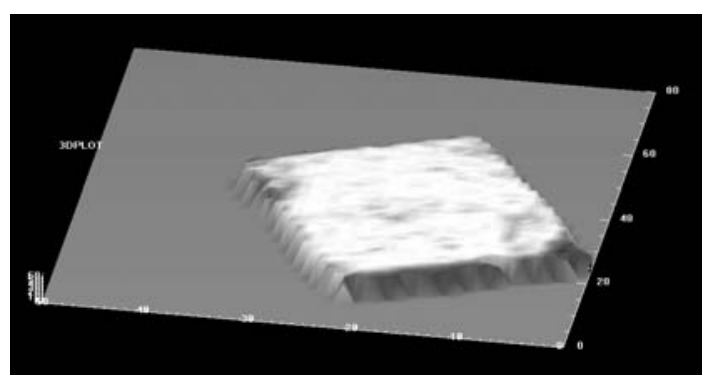

(b)

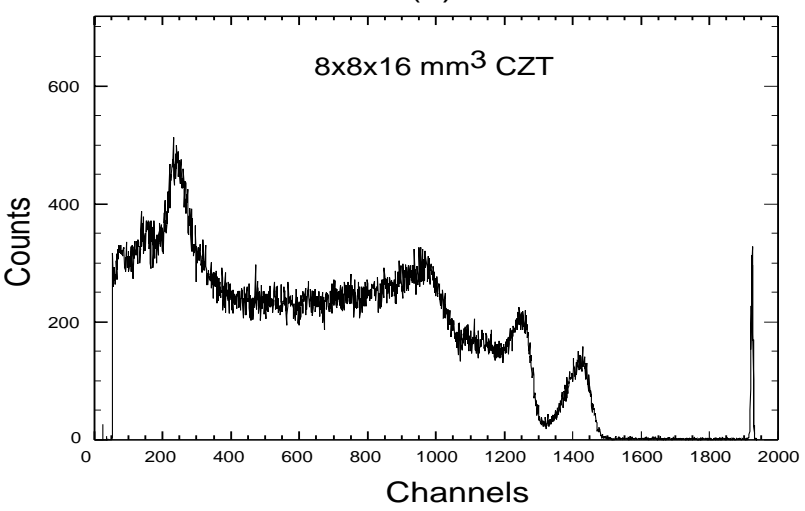

$X$-ray map

$8 \times 8 \times 16 \mathrm{~mm}^{3} \mathrm{CZT}$

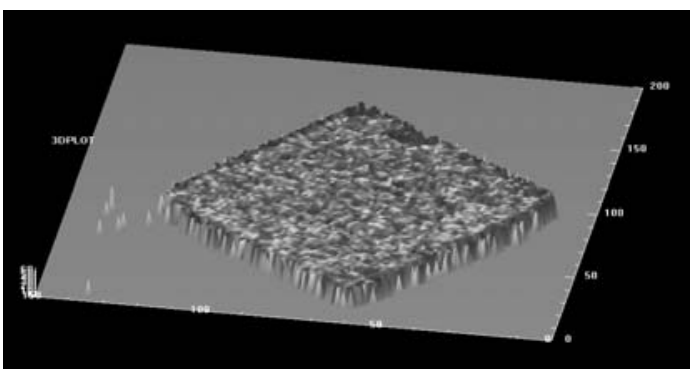

Fig. $1 .{ }^{60}$ Co spectra and X-ray scans measured for two Frisch-ring CZT detectors: (a) 11-mm long with a low concentration of Te precipitates, and (b) 16- $\mathrm{mm}$ long with high concentration of precipitates. The scans were performed by using a $25 \times 25 \mu^{2}$ size, $85-\mathrm{keV}$ beam. 

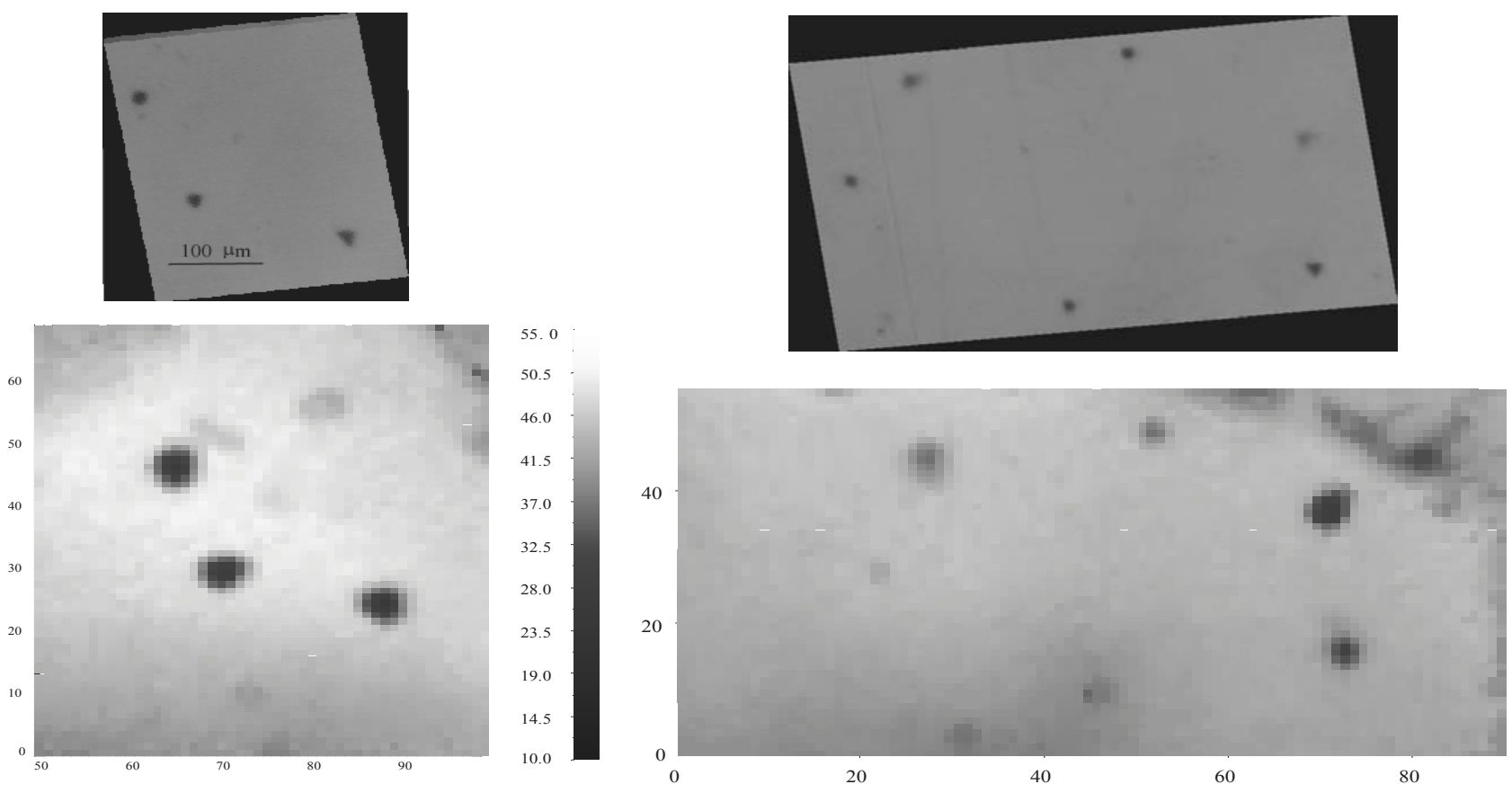

Fig. 2. Examples of correlations between X-ray and IR transmission maps measured for a 1-mm thick CZT planar device. The scans were performed by using a 10x10 $\mu \mathrm{m}^{2}$ size, $85-\mathrm{keV}$ X-ray beam. In some cases, the typical triangular shapes of precipitates are recognizable in the X-ray maps. 


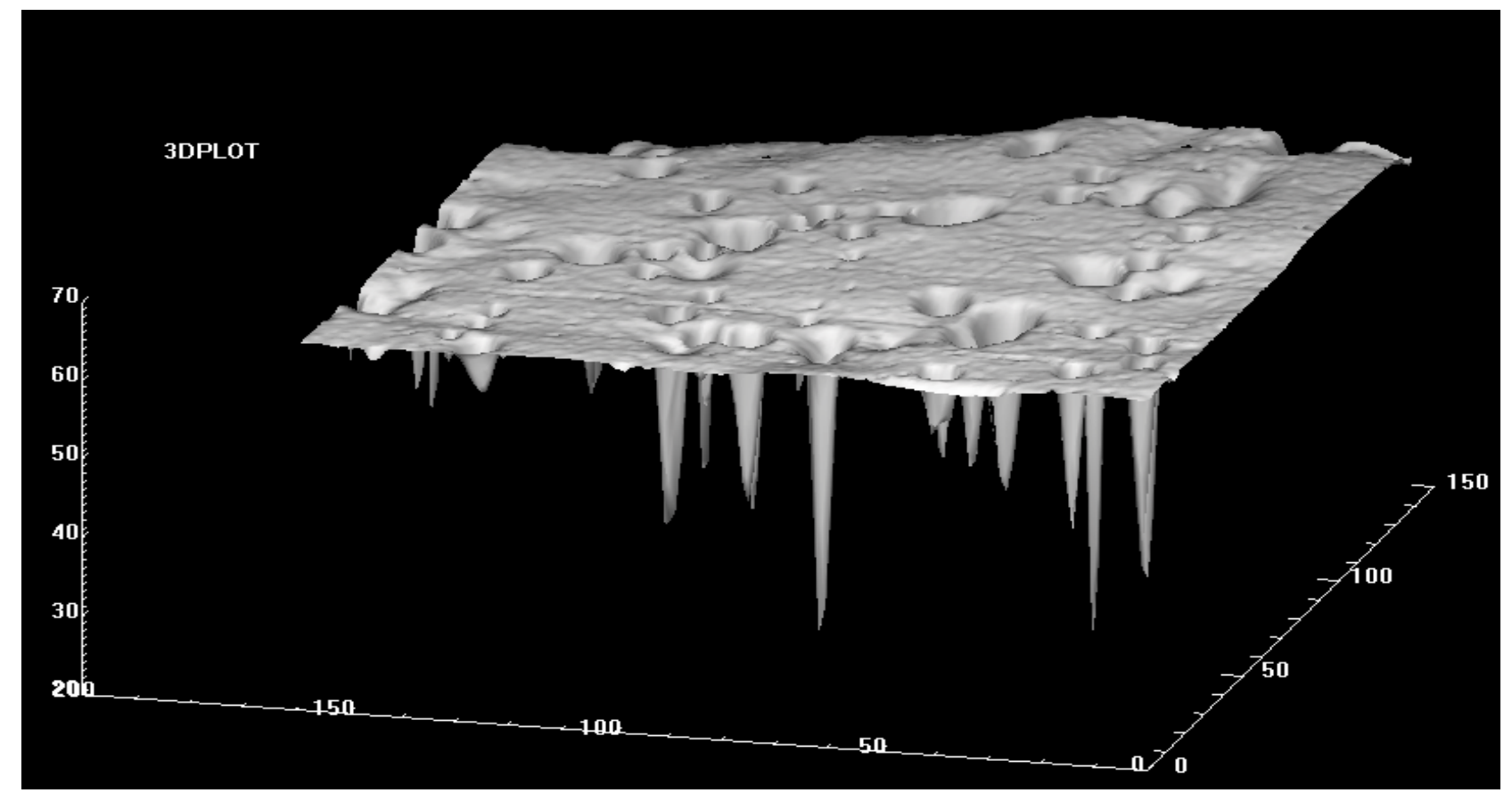

Fig. 3. Three-dimensional view of an X-ray map. The scan was performed by using a $10 \times 10 \mu \mathrm{m}^{2}$ size, 30-keV beam. 
$10 \mu \mathrm{m} \times 10 \mu \mathrm{m}$

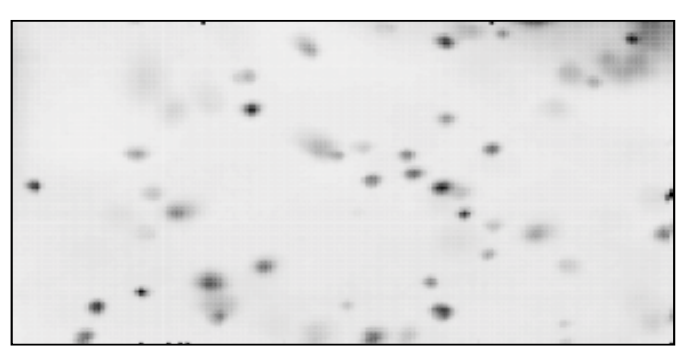

$100 \mu \mathrm{m} \times 100 \mu \mathrm{m}$

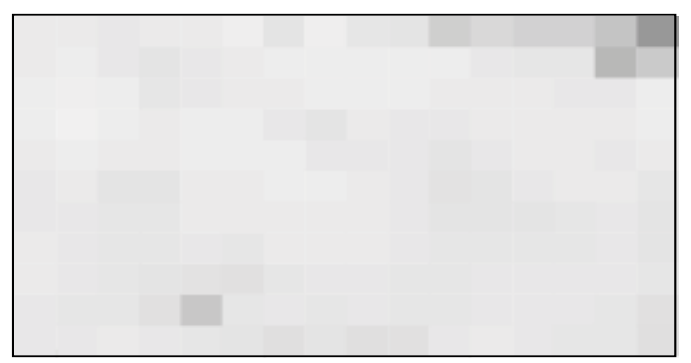

$20 \mu \mathrm{m} \times 20 \mu \mathrm{m}$

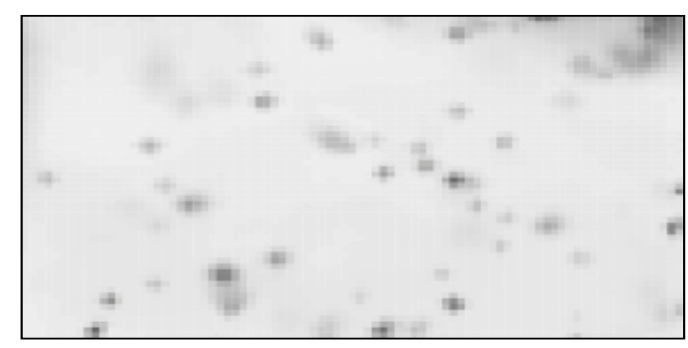

$200 \mu \mathrm{m} \times 200 \mu \mathrm{m}$

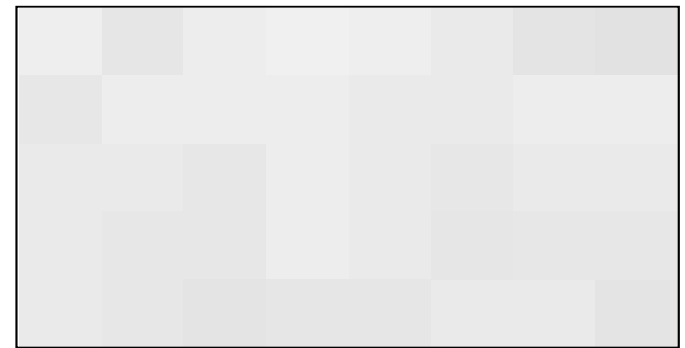

Fig. 4. X-ray maps evaluated for the same area of the device, but with different spatial resolution: $10 \times 10,20 \times 20,100 \times 100$, and $200 \times 200 \mu \mathrm{m}^{2}$. 
References:

[1] R. B. James, T. E. Schlesinger, J. C. Lund and M. Schieber, Semiconductors for Room Temperature Nuclear Detector Applications, Vol. 43 (Academic Press, New York, 1995), p. 334.

[2] C. Szeles and E. E. Eissler, Semiconductors for Room Temperature Radiation Detector Applications II, Vol. 487, (Materials Research Society, Pittsburgh, PA, 1998), p. 3.

[3] J. R. Heffelfinger, D. L. Medlin, and R. B. James, Semiconductors for Room Temperature Radiation Detector Applications II, Vol. 487, (Materials Research Society, Pittsburgh, PA, 1998), p. 33.

[4] Yinnel Tech, Inc., Internal report.

[5] V. Ivanov, L. Alekseeva, P. Dorogov, A. Loutchanski, Proc. IEEE-RTSD, Rome 2004.

[6] W. J. McNeil, D. S. McGregor, A. E. Bolotnikov, G. W. Wright and R. B. James, Appl. Phys. Lett. 84, No. 11, 1988 (2004).

[7] A. E. Bolotnikov, G. C. Camarda, G. A. Carini, M. Fiederle, L. Li, D. S. McGregor, W. McNeil, G. W. Wright, and R. B. James, to be published in IEEE Trans. Nucl. Sci., 2005.

[8] R. B. James, B. Brunett, J. Heffelfinger, J. Van Scyoc, J. Lund, F. P. Doty, C. L. Lingren, R. Olsen, E. Cross, H. Hermon, H. Yoon, N. Hilton, M. Schieber, J. Toney, T. E. Schlesinger, M. Goorsky, W. Yao, H. Chen, and A. Burger, J. Electron. Materials 28, 234 (1998).

[9] P.N. Luke, M. Amman, and J.S. Lee, IEEE Trans. Nucl. Sci. 51, 1199 (2004). 